\title{
Accelerators R\&D
}

\section{Caterina Biscari}

$L N F-I N F N$

Via Enrico Fermi 40, Frascati, 00044, Italy

E-mail: caterina.biscarielnf.infn.it

R\&D on particle accelerators dedicated to high energy physics applications are reviewed. Progress in particle colliders follows two different paths, one aiming at increasing the available energy in collision, the other at increasing the luminosity. Present collider performances and their plans on both fronts for the next future are described, together with the ideas and the technologies being developed for the projects that will be built after LHC. Neutrino beam facilities and plans for plasma wakefield accelerators are mentioned.

European Physical Society Europhysics Conference on High Energy Physics EPS-HEP 2009, 


\section{Introduction}

Particle accelerators in the world are several thousands. The largest number is dedicated to practical applications, mainly in industry and medicine. Accelerators dedicated to fundamental physics are a small fraction of the total number, but it is mainly in the laboratories where they are in operation that the most innovative ideas and challenging technologies are developed, tested, commissioned, and once they become fully reliable, exported to the practical world: technological advances go in parallel with the advances in knowledge of the physical laws which master our universe.

High energy physics is the engine which has driven the development of technologies for the acceleration of particle beams at higher and higher energies.

The history of colliders began almost fifty years ago, with the small prototype ADA (1), where for the first time electrons and positrons stored in an accelerator were colliding. During the last five decades collider energies and luminosities have increased by five orders of magnitude, as shown in Figure 1, where full symbols correspond to past or present accelerators, and empty ones to proposed projects for the future, none of which has been yet approved or funded. Presently only eight particle colliders are operational in the world, here including LHC (2). High energy protons collide in the Tevatron (3), ions and protons in RHIC (4), and four lepton factories, together with the old VEPP4M (5), complete the scenario of high energy physics with colliding beams.

Keeping in the future the same growing law of energy and luminosity as in the past is becoming more and more challenging. All proposals for future colliders are studied by international collaborations since they imply very complex systems, not fitting national sized laboratories. New acceleration techniques, like the plasma wakefield acceleration, are being developed in test facilities, with the prospect of enlarging the energy frontiers.

Accelerators are also used to produce intense beams of neutrinos, directed to underground detectors, complementary to the solar neutrinos detection.
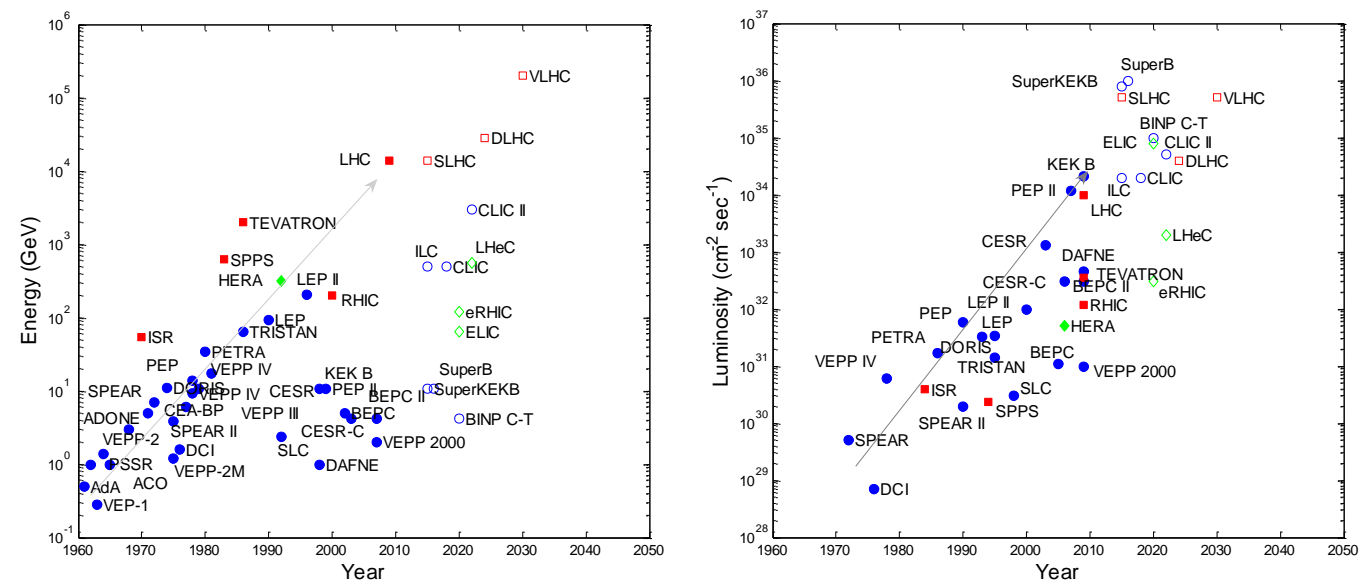

Figure 1. Energy and luminosity in particle collider history and future prospect. Full symbol: past and present projects. Empty symbols: future. Blue: leptons, Red: hadrons, Green: leptons-hadrons 


\section{Energy frontiers}

\section{Electron and positron colliders}

LEP (6) has been the last circular electron-positron collider at the high energy frontier. The $100 \mathrm{GeV}$ particles stored in LEP radiated 3\% of their total energy per turn via synchrotron radiation emission. Energy losses are proportional to the fourth power of energy and only directly proportional to the inverse bending radius; it is straightforward that next future collider will be linear.

Linear accelerator means high gradient rf cavities. Two technologies are being developed in parallel: ILC (7), based on superconducting (sc) technology and CLIC (8), based on two beam acceleration. The reachable energies in the center of mass are $1 \mathrm{TeV}$ for ILC and $3 \mathrm{TeV}$ for CLIC. Both projects foresee an initial phase at $0.5 \mathrm{TeV}$.

The decision to use cold technology for $I L C$ was taken in 2004, by the International Technology Recommendation Panel, and one of the strongest reasons was the well advanced R\&D on the TESLA (9) technology at $1.3 \mathrm{GHz}$. Since then, under the GDE (Global Design Effort) coordination, an effort on the three regions (America, Asia and Europe) is being done to achieve the design performances, with a large involvement of vendors. The FLASH facility (10) at DESY, routinely providing photon beams to users since 2007, is the most advanced test facility for the sc cavities and related systems. XFEL (11), the international project for coherent $\mathrm{X}$ ray production at $10 \mathrm{GeV}$, being built at DESY, will provide a $10 \%$ scaled ILC system.

Cavities are built and tested in the three world regions, which are expected to contribute to the construction of the main linacs, irrespective of where the machine will be built. The goal is to 'qualify' at least two industrial vendors in each region. In addition, the technology has wide applicability beyond ILC which makes it highly-attractive to national funding agencies.

The nominal accelerating gradient is $31.5 \mathrm{MV} / \mathrm{m}$. It has been achieved and surpassed in many cases (see Figure 2). In view of the mass production the key parameter is the yield of cavities reaching the nominal design parameters. Present $R \& D$ aim is demonstrating by the end of 2010 a process yield of $50 \%$ and by the end of 2012 a production yield of $90 \%$ at $35 \mathrm{MV} / \mathrm{m}$. Up to now an average of 48 tests on 19 cavities have been done and almost $60 \%$ of them have surpassed the nominal gradient. In particular one of the vendors has tested 11 cavities and more than $50 \%$ have achieved $35 \mathrm{MV} / \mathrm{m}$.
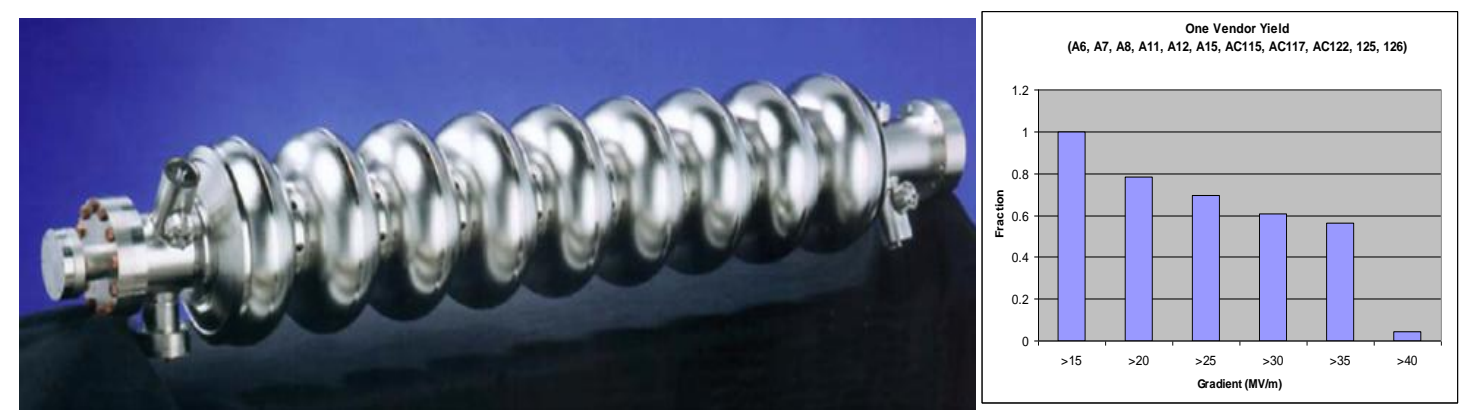

Figure 2. ILC SC rf cavity and one vendor yield 
A primary identified gradient limitation is the field emission, lately significantly reduced with the application of so called degreasing techniques after electro-polishing. Focus has now shifted to consolidating mechanical fabrication. Novel high-resolution optical inspection techniques have revolutionized the analysis of the equator weld and 'heat affected' zone. They are being developed with the goal to establish the technique as part of industrial production.

Another R\&D priority for the main linac technology is the production of a cryomodule with an average accelerating gradient of $31.5 \mathrm{MV} / \mathrm{m}$. A GDE-driven international collaboration, hosted by KEK, will attempt to construct a single cryomodule with eight high-gradient cavities supplied by KEK, DESY and FNAL with different designs. KEK's responsibility is to provide the mechanical integration into a single unit.

The dual beam acceleration technology was proposed almost twenty years ago (12), and three CLIC test facilities have been built at CERN during these years in order to test the feasibility of this solution. Main issues to be demonstrated are the high accelerating gradient of main linac, the production of the drive beam and the power transmission.

The main limitation to the accelerating field in copper cavities is given by rf breakdowns, the maximum breakdown rate required for stable beam operation in a multi-TeV linear collider being in the range of a few $10^{-7}$ per meter. The improved knowledge of this phenomenon, combining results from rf structure testing and optimization studies of overall cost and performance, led at the beginning of 2007 to a major parameter revision for CLIC. The CLIC rf frequency was lowered from $30 \mathrm{GHz}$ to $12 \mathrm{GHz}$ and its accelerating gradient reduced from 150 $\mathrm{MV} / \mathrm{m}$ to $100 \mathrm{MV} / \mathrm{m}$.

Recent experimental tests confirmed that such a gradient is accessible, at the required pulse length and breakdown rate: thanks to the collaboration between KEK, SLAC and CERN a CLIC-like accelerating structure has been successfully tested (see Figure 3) obtaining an unloaded gradient above $100 \mathrm{MV} / \mathrm{m}$ with the nominal pulse length and a breakdown probability of about $3 \times 10^{-7} / \mathrm{m}$ corresponding to CLIC specifications. The CERN design was built in KEK and sent to SLAC for assembly and RF testing. This test structure does not yet incorporate the needed damping features. The full demonstration of a CLIC structure with damping is presently under preparation. The next step will be facing the mass production organization, assuring the production yield of structures with the nominal parameters.
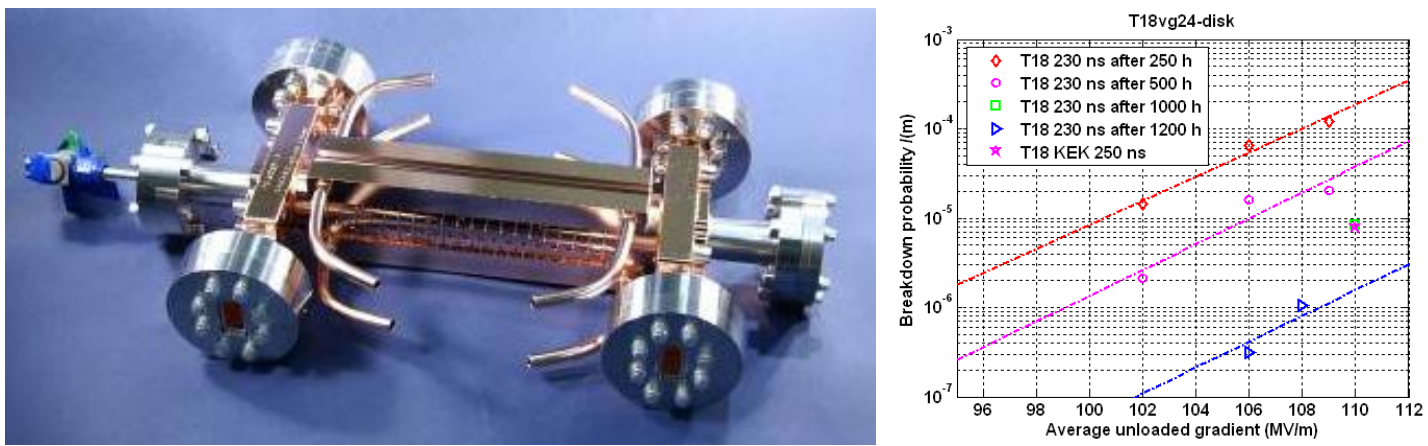

Figure 3. CLIC-like accelerating structure T18 vg2.4 disk, and its performance in term of gradient and rf breakdown rate. 
In CLIC the rf power is extracted from a high current - low energy drive beam. Its bunch frequency structure allows the production of rf power at the desired frequency, by decelerating it through the Power Extraction and Transfer Structures (PETS). Each PETS extracts 136 MW of RF power and feeds it into two accelerating structures in the main linac.

The CLIC Test Facility at CERN (CTF3), presently in commissioning at CERN under an international collaboration, is a scaled-down version of the drive beam generation system, at lower energy (150 MeV instead of $2 \mathrm{GeV}$ ), and shorter pulse lengths (few hundreds of ns instead of $140 \mu \mathrm{sec}$ ). It has already produced a series of results (13): validation of the drive beam generation scheme with a fully-loaded linac RF-to-beam energy transfer efficiency of 95\%, including structure losses; sub-harmonic bunching system for a fast RF phase switch of $180^{\circ}$ and beam current recombination factor of the beam pulses by a factor of 2 in the Delay Loop; recombination by a factor of 4 in the Combiner Ring, where currents of $15 \mathrm{~A}$ have been obtained (see Figure 4), together with the precise determination of the ring length to the submillimetre level as required; design and construction of damped PETS, which can be switched on and off, tested with a 5A beam in the TBTS (Two Beam Test Stand), using a recirculation concept which highly amplifies its output, obtaining up to 30MW.

The next future program includes the full recombination using both DL and CR, aiming at a total current of 30A, the study of drive beam deceleration in a string of PETS, the assessment of the drive beam stability in current and phase, and the implementation of a fast phase feedback system in order to demonstrate the very tight phase stability (better than 0.2 degrees at 12 $\mathrm{GHz}$ ) required for the CLIC drive beam.

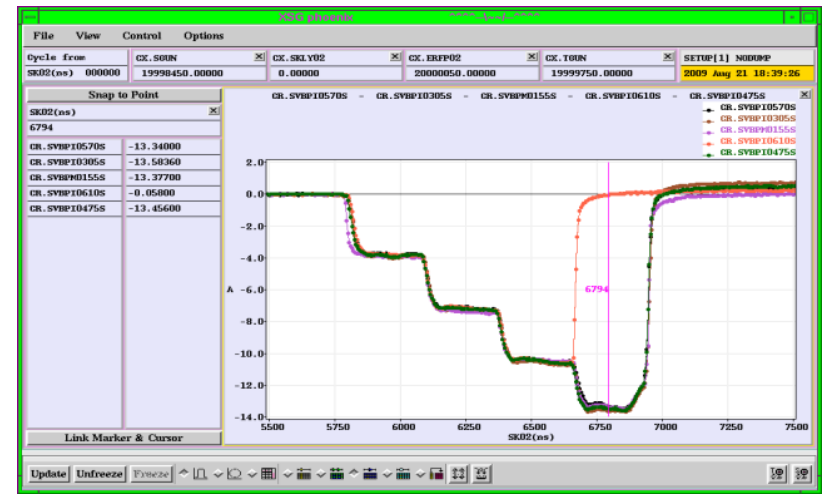

Figure 4. Recombination by a factor of four in the CTF3 combiner Ring. The beam current as a function of time in the first two Beam Position Monitors is shown.

The two LC communities have worked in parallel and in constructive competition for years, with an always increasing collaboration on common subjects.

This collaboration has recently been officially stated, with the aim of making the best use of the available resources. The aim is adopting as similar as possible systems, identifying and understanding the differences due to technology and energy, developing common knowledge of both designs and technologies on status, advantages, issues and prospects for the best use of the future LC. CLIC \& ILC will prepare together the future evaluation of the two technologies, defining which proposal is best adapted to the future HEP requirements. 


\section{Hadron colliders}

While the energy frontier in lepton colliders is mainly driven by the rf accelerating gradients, high magnetic field magnets are the critical element for hadron colliders energy frontier. Presently Tevatron (14) is the highest energy collider, with its $1 \mathrm{TeV}$ beams.

When LHC will come into operation next fall (15) it will run for the first period at an energy per beam of $3.5 \mathrm{TeV}$, half of its maximum energy. The main reason of this conservative approach is the incident occurred in the past year, and the necessary caution to deal with the complicated system which assures the reliability of the whole accelerator complex. The LHC maximum dipole field is presently $8.3 \mathrm{~T}$, obtained using Niobium-Titanium (NbTi) conductor at $1.9 \mathrm{~K}$.

One of the foreseen LHC upgrades is doubling its present maximum energy (DLHC) (16), implying the substitution of magnets by higher field ones. $R \& D$ on new materials is driven also by LHC luminosity upgrades, where very high gradient quadrupole doublets in the Interaction Regions (IRs) will be used. Much higher energies would be those involved in the ultimate LHC upgrade, TLHC, with 3 times the present design energy, and the US proposal of VLHC, the Very Large Hadron Collider, whose energy in the center of mass should reach $400 \mathrm{TeV}$ (17).

R\&D for high magnetic fields (18) addresses the developments in sc materials, to achieve better efficiency and simplify magnet fabrication while preserving accelerator-class field quality. Niobium-Tin $\left(\mathrm{Nb}_{3} \mathrm{Sn}\right)$ is the most promising material, and it has already been used for dipole prototypes. It carries current densities similar to $\mathrm{NbTi}$ at more than twice the field and at $4 \mathrm{~K}$. It is available in long samples with uniform properties, and it is expected that in few years from now the technology will be mature enough for regular production of magnets.

Because of their brittleness, high field superconductors cannot be drawn to thin filaments like $\mathrm{NbTi}$, but have to be formed in the final geometry by high-temperature heat treatment. In the fully reacted state, the filaments are extremely sensitive to strain. An adequate coil support requires special mechanical designs, to minimize the stress on the conductor at all stages of magnet fabrication and operation.

The ultimate magnetic field in a practical dipole configuration using $\mathrm{Nb}_{3} \mathrm{Sn}$ technology is limited to 17-18 T by the material's maximum critical field $\mu_{\mathrm{o}} \mathrm{H}_{\mathrm{c} 2}$ of about $29 \mathrm{~T}$. In order to develop dipoles approaching $20 \mathrm{~T}$ and higher, a material with a higher $\mathrm{H}_{\mathrm{c} 2}$ is required (see Figure 5).

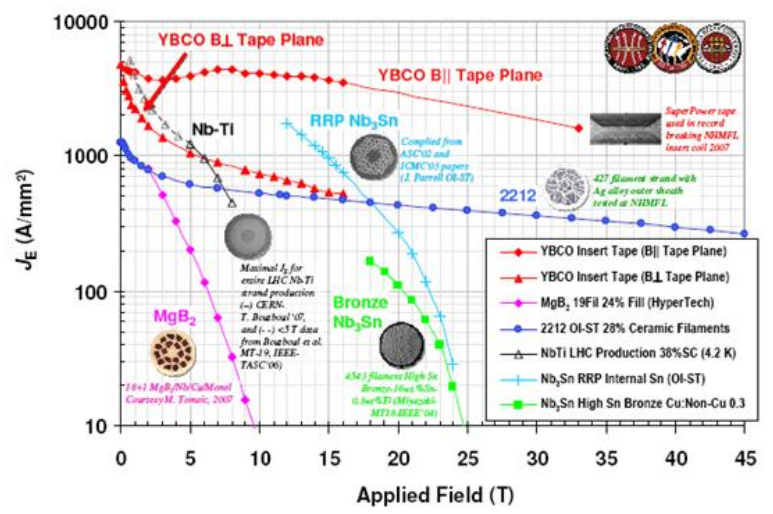

Figure 5. Current density in SC materials as a function of the applied magnetic field. 
The most promising candidate for this purpose is $\mathrm{Bi}-2212$, which is available in round wires and has been made in sufficient lengths for the fabrication of coils based on Rutherfordtype cables. The new technological challenges are related to cabling of the wires, higher strain sensitivity, high formation reaction temperature in an oxygen-rich environment, and chemical compatibility of the insulation and construction materials during the reaction heat treatment. However, many fundamental technology issues need to be addressed before practical magnet designs can be developed and implemented in prototypes.

\section{Luminosity frontiers}

We can summarize the challenge of high luminosity as the capability of keeping small beam sizes in high charge bunches, with high repetition frequency, while fighting against the beam-beam effects. Luminosity frontiers are driven by intensity and beam size frontiers.

In collider history the increase in luminosity has often been related to the increase in particle energies. This trend does not apply to lepton factories, where luminosity frontier has been pushed keeping beam energy at particle resonance values $(\Phi$, tau, B), as can be seen in Figure 6, where collider luminosities are plotted versus energy: as an example it is worth remarking that DAФNE, the Frascati $\Phi$-factory ( $1 \mathrm{GeV}$ energy in the center of mass), has reached a higher luminosity than LEP.

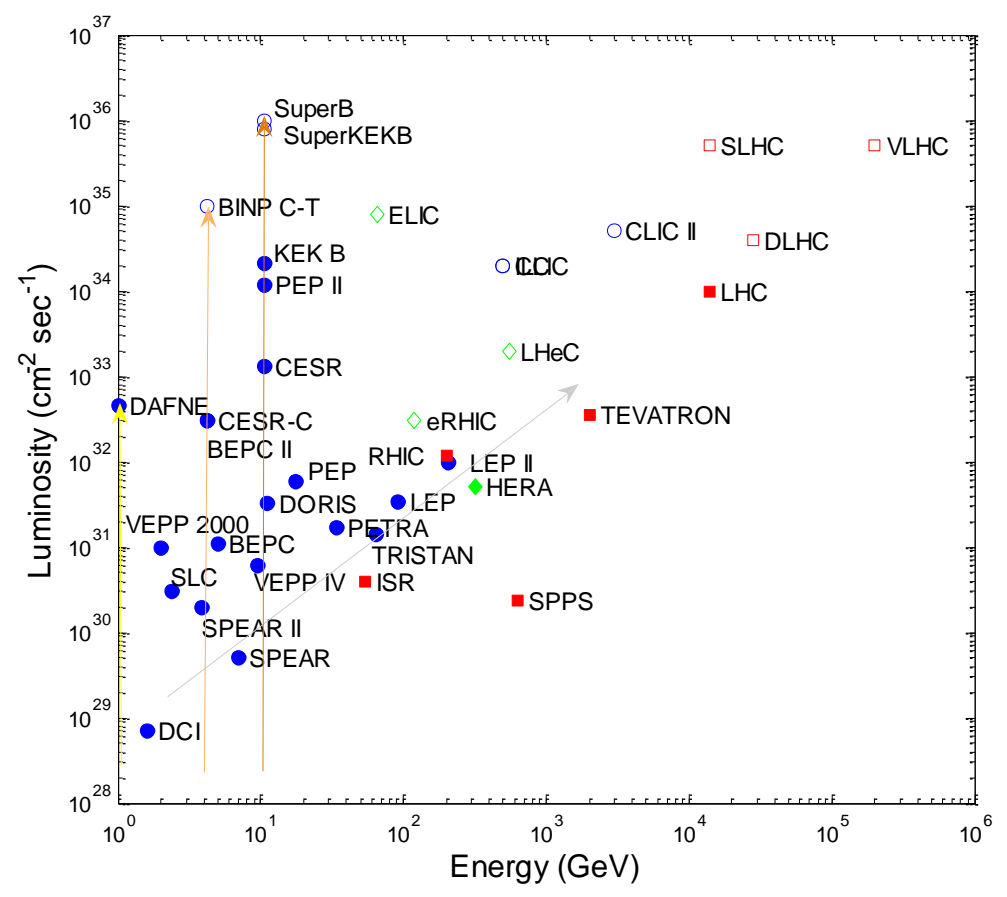

Figure 6. Luminosity versus energy of colliders. Symbols have same meaning of Figure 1.

Let's describe shortly the approaches for reaching high luminosities in the operating colliders and in the future projects, differentiating among the collider type. 


\section{Lepton factories}

Four lepton factories are presently in operation in the world, working at different energies. Each of them is based on a particular collision scheme. Table I shows a list of their main parameters. The main challenges for lepton factories have proven to be high circulating currents, fighting against instabilities and collective effects, and beam-beam effects. The new schemes are all meant to weaken beam-beam effects, in order to increase luminosity without pushing further bunch charges.

Impressive development of feedback systems, optimization of rf cavities, vacuum chamber and diagnostics designs minimizing impedances, have allowed to store in the factories rings very high currents: the maximum current ever stored in an electron ring is the $2.4 \mathrm{~A}$ in DAФNE while PEP II holds the record for positrons: up to 3A.

A comprehensive and up to date overview of the lepton factories main achievements and future prospects can be found in ref (19).

BEPC II (20) - classical scheme.

The tau-charm factory of Beijing is based on what can be now considered the classical scheme for lepton factories: double ring high current multibunch operation, high collision frequency, small crossing angle. It was the scheme adopted in the late nineties by DAФNE (21), PEPII (22) and KEK-B (23), which has proven to be effective in obtaining high rate of collisions.

BEPC II has now completed its first commissioning phase, begun in May 2008, and has already reached a peak luminosity of $3.310^{32} \mathrm{~cm}^{-2} \mathrm{sec}^{-1}$, corresponding to $30 \%$ of its design value, limited by the storable current. In the present shutdown, feedback systems are being installed, in order to increase the beam currents, and correspondingly the luminosity.

\section{VEPP 2000 (24) - round beam.}

In Novosibirsk the round beam collision scheme is being tested in VEPP 2000, a $24 \mathrm{~m}$ long ring, which can be operated at energies in the center of mass between 1 and $2 \mathrm{GeV}$. The ring works on the main coupling resonance, with equal horizontal and vertical emittances and tunes. During the first commissioning phase at the lowest energies, in single bunch mode, a luminosity of $10^{31} \mathrm{~cm}^{-2} \mathrm{sec}^{-1}$ has been reached, which corresponds to the highest single bunch luminosity at this energy, and to a very high beam-beam tune shift, as predicted by round-beam collision simulations. Potentially it should reach twice as much in the low energy regime and up to $1.610^{32} \mathrm{~cm}^{-2} \mathrm{sec}^{-1}$ at $2 \mathrm{GeV}$.

\section{DA $\Phi N E-c r a b$ waist}

$D A \Phi N E$, the Frascati $\Phi$-factory, has been operated during ten years, delivering luminosity to three different experiments, which have been alternatively installed in one of the two IPs. With the classical scheme it has reached a luminosity of $1.610^{32} \mathrm{~cm}^{-2} \mathrm{sec}^{-1}$, with maximum colliding currents of $\sim 2 \mathrm{~A}$ in the electron ring and $\sim 1 \mathrm{~A}$ in the positron one.

During a shutdown in 2007 the crab waist scheme (25) has been implemented in the collider and it is being tested with the experiment SIDDHARTA, which does not use any 
magnetic field for particle detection. From 2010 on the main DAФNE experiment, KLOE, will be reinstalled in the collider, with a new IR layout to compensate the detector solenoidal field.

In the crab waist (CW) scheme beams collide with a large Piwinski angle (LPA), thanks to very low horizontal emittance, long bunches and large crossing angle. The overlap area of colliding bunches is reduced to a small spot size around the IP, and higher luminosity can be reached. There are additional advantages in such a collision scheme: there is no need to decrease the bunch length to increase the luminosity thus reducing the problems of HOM heating, coherent synchrotron radiation of short bunches, excessive power consumption, etc... Moreover the problem of parasitic collisions (PC) is automatically solved by the higher crossing angle and smaller horizontal beam size, which makes the beam separation at the PC large in terms of horizontal beam sizes, with a reduction of the vertical tune shift.

The idea of colliding with a large Piwinski angle is not new (see for example (26)). It has been also proposed for the LHC upgrade to increase the bunch length and the crossing angle.

However, a LPA itself introduces new beam-beam resonances and may strongly limit the maximum achievable tune shifts. This is where the crabbed waist innovation is required. A pair of sextupoles on the IP sides, in phase with the IP in the horizontal plane and at $\pi / 2$ odd multiples in the vertical plane, correlate the particle position with the vertical $\beta$ waist, so that all particles cross the maximum density of the other beam at their minimum vertical $\beta$, (see Figure 7). The crabbed waist transformation boosts the luminosity, mainly by suppression of betatron and synchro-betatron resonances.

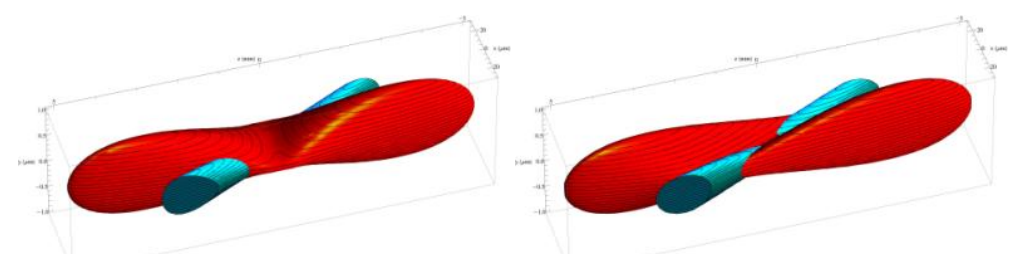

Figure 7. Crab waist collision scheme representation: crab sextupoles off(left), on (right).

In DAФNE the peak luminosity with the crab waist collision scheme has reached $4.510^{32}$ $\mathrm{cm}^{-2} \mathrm{sec}^{-1}$ with circulating currents of $\sim 2 \mathrm{~A}$ electrons and $\sim 1.2 \mathrm{~A}$ positrons, almost a factor of tree on the previous result, with a maximum integrated luminosity per day of 15 pbarn $^{-1}, 50 \%$ increase with respect to the classical scheme.

The crab waist scheme, also thanks to the success of the DAФNE test, is being now considered in many of the future upgrade projects of different colliders. It is furthermore the basis of the SuperB project, a proposal for building nearby Rome a B-factory (27), with unprecedented luminosity, $10^{36} \mathrm{~cm}^{-2} \mathrm{sec}^{-1}$, and of the BINP-tau charm proposal (28).

In SuperB the LPA\&CW scheme, together with very low $\beta^{*}$, will allow for operation with relatively low beam currents and reasonable bunch length, comparable to those already used in PEP-II and KEK-B. In the electron ring spin rotators will provide longitudinal polarized beams, this being an unique feature for a factory. The ring lattice has been designed with a very low intrinsic emittance and is quite compact, less than $2 \mathrm{~km}$ long. Special designs are being proposed for the final doublet IR quadrupoles, which are very close to the IP, to avoid excessive background from synchrotron radiation. 
A Conceptual Design Report, written by an international collaboration, was published in March 2007 (27), and R\&D studies are in progress in order to publish a Technical Design Report by the end of 2010. The most relevant parameters are listed in Table I.

\section{KEK-B - crab cavities.}

KEK-B (29) has been, together with PEP-II (30), the B-factory operating in the last decade, which has reached performances and luminosity above design value. Its basic features are the same of the above mentioned DAФNE and BEPC-II. With the classical scheme KEK-B reached in 2006 a peak luminosity of $1.710^{34} \mathrm{~cm}^{-2} \mathrm{sec}^{-1}$, the highest luminosity ever obtained in any collider (see Figure 8).

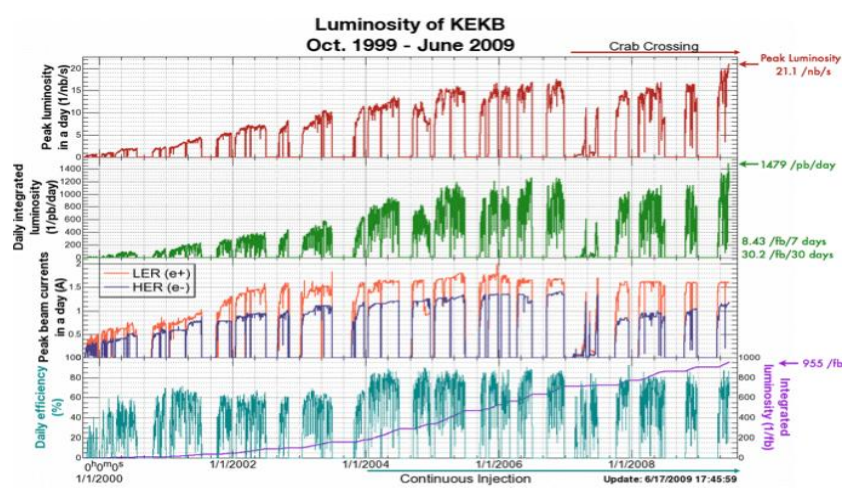

Figure 8. KEK-B luminosity history

The collision scheme which is being tested nowadays in KEK-B is the crab crossing, which, in spite of the linguistic similarity, is very different from the crab waist. It is based on if cavities (crab cavities), placed along the ring, giving a transverse kick which correlates horizontal and longitudinal plane, producing an oscillation of the bunch around its center, with the phase advance and kick amplitude such that when beams arrive at the IP, they collide headon avoiding the crossing angle effects (see Figure 9). Two cavities per ring is the optimum configuration, so that beams oscillate only in between the two cavities.

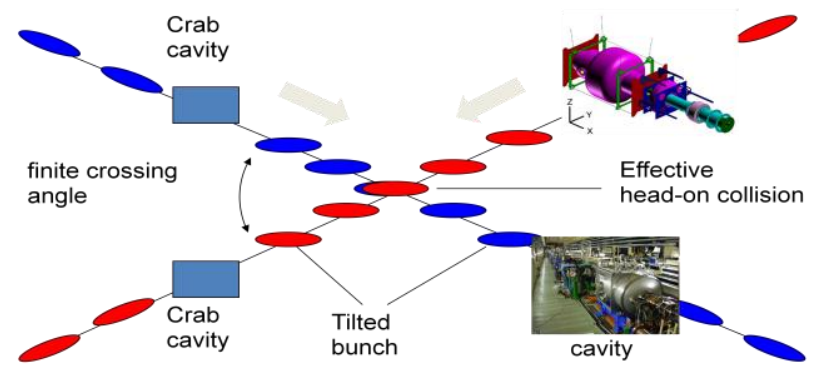

Figure 9. Crab cavity collision representation

During a shutdown in 2007 one crab cavity was installed in each ring in order to test the principle and to boost the luminosity. This scheme produces oscillations all along the ring. Restoring the luminosity level obtained before the upgrade has not been straightforward. Lately 
during this year a significant improvement of performances has been obtained by fine tuning all collider parameters, in particular the betatron coupling in both rings. The peak luminosity so obtained, $2.110^{34} \mathrm{~cm}^{-2} \mathrm{sec}^{-1}$, is the highest ever achieved. The daily integrated luminosity is twice as high as the design due to the Continuous Injection Mode as well as acceleration of two bunches per rf pulse at the linac. The electron cloud in the LER, which was much more severe than expected, has been mitigated up to $1.8 \mathrm{~A}$ with 3 bucket spacing and solenoid windings along the ring. During these ten years of KEK-B operation the total integrated luminosity is almost 1 abarn $^{-1}$.

Upgrade plans for increasing the luminosity by a factor of 50 are been considered in the SuperKEKB project. One is based on very high currents (9.4 and 4.1 A), crab cavities, small $\beta^{*}$ and very short bunches. The main issues of such approach are all associated with the high currents. Simulations of the electron cloud effects and of the bunch length increase in LER by coherent synchrotron radiation indicate that severe limits are posed on the maximum obtainable luminosity.

A second approach, called Nano-Beam Scheme, seems to promise higher luminosity with less risks. It is based on the same concept of the above mentioned SuperB, with very low emittance beams, crossing with a higher Piwinski angle, longer bunches, and lower currents (3.3 and $1.9 \mathrm{~A})$. The main challenge of such a scheme is keeping the beam stability control of collision with tiny beam size and emittance preservation against vibration and drift in the ring components.

A decision on which approach will be followed will be taken during this year.

Table I - Present and proposed lepton factories main parameters

\begin{tabular}{|c|c|c|c|c|c|c|c|}
\hline & & 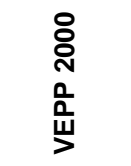 & 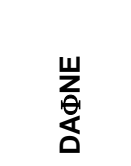 & $\begin{array}{l}\overline{\bar{j}} \\
\text { 㟧 }\end{array}$ & $\begin{array}{l}\underset{\boldsymbol{q}}{\mathbf{u}} \\
\underset{\underline{x}}{ }\end{array}$ & 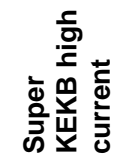 & $\begin{array}{l}\frac{\infty}{d} \\
\text { 윽 } \\
\text { ஸ }\end{array}$ \\
\hline Energy $(L / H)$ & $\mathrm{GeV}$ & $0.5-1$. & $0.51 / 0.51$ & $1.9 / 1.9$ & $3.5 / 8$ & $3.5 / 8$ & $4 / 7$ \\
\hline Luminosity & $\mathrm{cm}^{-2} \mathrm{sec}^{-1}$ & $1.110^{31}$ & $4.210^{32}$ & $3.310^{32}$ & $2.110^{34}$ & $810^{35}$ & $1.010^{36}$ \\
\hline C & $\mathrm{m}$ & 24 & 97 & 238 & 3016 & 3016 & 2000 \\
\hline I (L/H) & $A$ & 0.4 & $2 . / 1.2$ & $0.9 / 0.9$ & $2.4 / 2$ & $9.4 \times 4.1$ & $2.8 \times 2.8$ \\
\hline $\mathrm{N}_{\text {bunches }}(\mathrm{L} / \mathrm{H})$ & & 1 & 105 & 93 & 1700 & 5000 & 2400 \\
\hline$\varepsilon_{y}^{*}(\mathrm{~L} / \mathrm{H})$ & $\mathrm{nm}$ rad & 130. & .70 & 1.4 & $240 / 90$ & $240 / 90$ & $7 / 4$ \\
\hline$\varepsilon_{\mathrm{x}}^{*}(\mathrm{~L} / \mathrm{H})$ & $\mu \mathrm{m}$ rad & 0.13 & 0.26 & 0.14 & $24 / 18$ & $24 / 18$ & $2.8 / 1.6$ \\
\hline$\beta_{y}{ }^{*}(L / H)$ & $\mathrm{cm}$ & 5 & 0.9 & 1.6 & 3. & 3. & $0.21 / 0.37$ \\
\hline$\beta_{x}{ }^{*}(L / H)$ & $\mathrm{cm}$ & 5 & 25. & 100 & 20. & 20. & $3.5 / 2.0$ \\
\hline Crossing angle & mrad & 0 & 40 & 22 & 30. to 0 . & 30. to 0 . & 60. \\
\hline Tune shifts $(L / H)$ & & 0.08 & 0.044 & 0.04 & $0.3 / 0.51$ & $0.3 / 0.51$ & $0.09 / 0.09$ \\
\hline
\end{tabular}




\section{Linear colliders}

Luminosity of both LC projects is in the range of $10^{34} \mathrm{~cm}^{-2} \mathrm{sec}^{-1}$. The issues to which most of the research is being addressed are the generation and preservation of the main beam low emittances, active alignment and stabilization of main and Final Doublet quadrupoles, focusing to nanometer dimensions the colliding beams. This is specially demanding for CLIC, where a stabilization of down to $0.15 \mathrm{~nm}$ above $4 \mathrm{~Hz}$ is needed to assure the right level of luminosity.

The low emittance beams are generated in the damping rings at low energies (few $\mathrm{GeVs}$ ) and again the CLIC parameters correspond to unprecedented low normalized equilibrium emittances of $3.3 \mathrm{~nm}$ vertical and $550 \mathrm{~nm}$ horizontal. The dominant emittance growth mechanism is intra-beam scattering, counteracted by intense synchrotron radiation damping from wigglers. Overall optimization of the wiggler parameters, taking into account state-of-theart wiggler technologies, wiggler effects on dynamic aperture, and problems of radiation absorption is being addressed, by investigating two technical solutions, one based on superconducting magnet technology, the other on permanent magnets. Touschek losses, electron cloud and fast ion instabilities are the other issues to be controlled for storing high currents in the damping rings.

Table II lists the main parameters of both LC options. Figure 10 and Figure 11 represent their schematic layout.

Table II - Linear colliders main parameters

\begin{tabular}{|c|c|c|c|}
\hline Center of-mass energy & ILC $500 \mathrm{GeV}$ & CLIC $500 \mathrm{GeV}$ & CLIC $3 \mathrm{TeV}$ \\
\hline Total (Peak $1 \%$ ) luminosity $10^{34}$ & $2.0(1.5)$ & $2.3(1.4)$ & $5.9(2.0)$ \\
\hline Repetition rate $(\mathrm{Hz})$ & 5 & 50 & 50 \\
\hline Loaded accel. gradient MV/m & 31.5 & 80 & 100 \\
\hline Main linac $\mathrm{RF}$ frequency $\mathrm{GHz}$ & 1.3 & 12 & 12 \\
\hline Bunch charge $10^{9}$ & 20 & 6.8 & 3.72 \\
\hline Bunch separation ns & 176 & 0.5 & 0.5 \\
\hline Beam pulse duration (ns) & 1000 & 177 & 156 \\
\hline Beam power/linac (MWatts) & 10.2 & 4.9 & 14 \\
\hline Hor/Vert norm. emit $\left(10^{-6} / 10^{-9}\right)$ & $10 / 40$ & $2.4 / 25$ & $0.66 / 20$ \\
\hline Hor/Vert FF focusing (mm) & $20 / 0.4$ & $8 / 0.1$ & $4 / 0.07$ \\
\hline Hor/Vert IP beam size $(\mathrm{nm})$ & $640 / 5.7$ & $202 / 2.3$ & $40 / 1.0$ \\
\hline BDS length (km) & 2.23 & 1.87 & 2.75 \\
\hline Total site length (km) & 31 & 13.0 & 48.3 \\
\hline Wall plug to beam transfer eff. & $9.4 \%$ & $7.5 \%$ & $6.8 \%$ \\
\hline Total power consumption MW & 216 & 129.4 & 415 \\
\hline
\end{tabular}




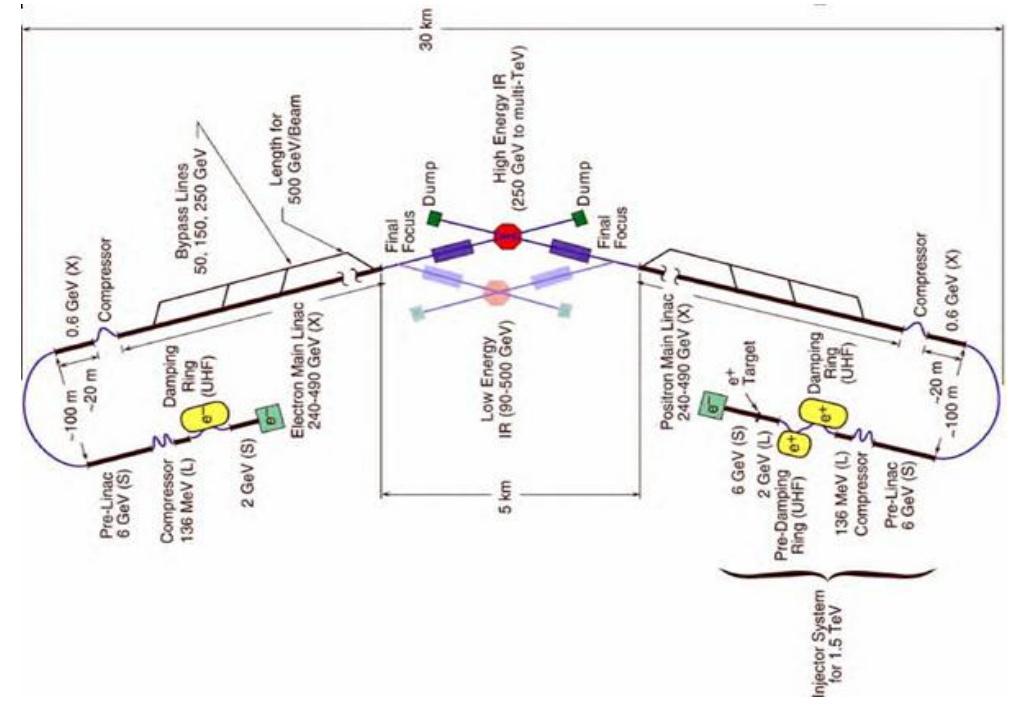

Figure 10. ILC schematic layout

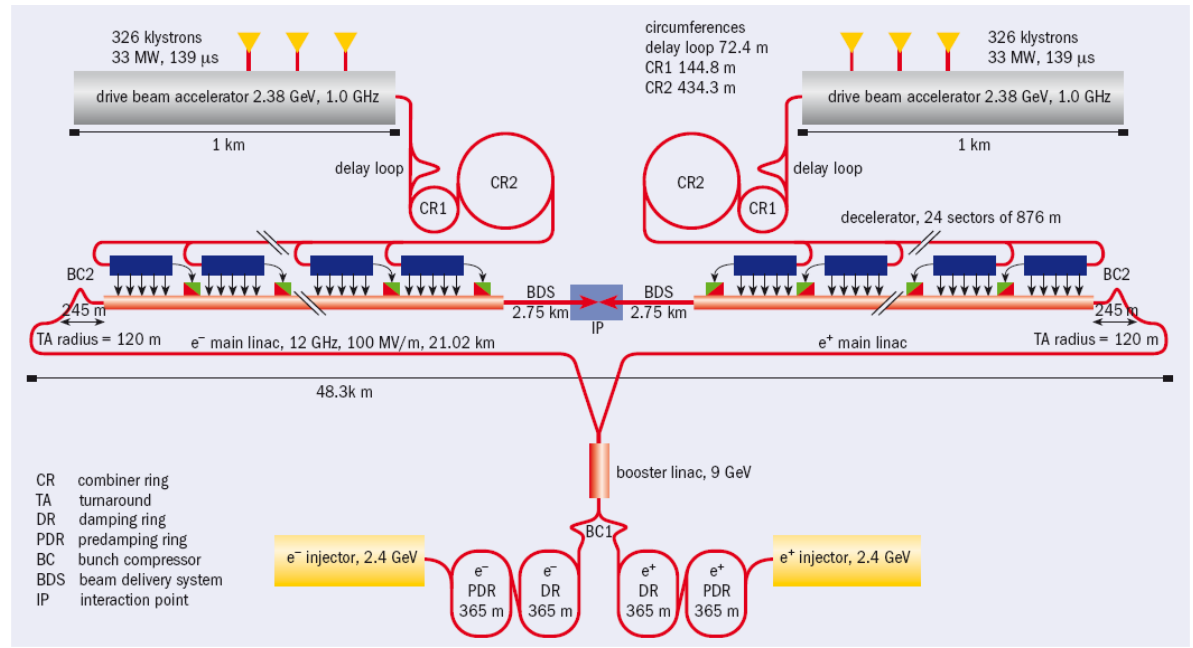

Figure 11. CLIC schematic layout

Test facilities are used to meet the critical LC challenges and validate simulation results. The CESR ring at Cornell is being transformed in CesrTA (CESR Damping Ring Test Accelerator) (31), where an aggressive program for the electron-cloud effect study is foreseen. ATF (Accelerator Test Facility at KEK) (32) is dedicated to the production of stable and very low emittances. It holds in fact the world record for the smallest measured emittance (33). Fast kickers similar to those needed for damping ring injection and extraction are tested there, as well as in DAФNE. ATF2 (34) is a final-focus test beam line that aims to focus the lowemittance beam from the ATF damping ring to a beam size of about $37 \mathrm{~nm}$, and at the same time to demonstrate $\mathrm{nm}$ beam stability, using several advanced beam diagnostics and feedback tools. ATF2 has been constructed and is being commissioned by ATF international collaborations with US, Asian and European participation. 


\section{Hadron colliders}

The maximum hadron collider luminosity has been reached by the Tevatron (14), with its peak luminosity of $3.1510^{32} \mathrm{~cm}^{-2} \mathrm{sec}^{-1}$, achieved during Run II (see Figure 12), at the center of mass energy of $1.96 \mathrm{TeV}$. The Tevatron is expected to deliver $\sim 6 \mathrm{fb}^{-1}$ per experiment by the end of fiscal year 2009 and, if it runs longer, $7-9 \mathrm{fb}^{-1}$ by the end of fiscal year 2010. Its final shutdown date will be defined after first LHC results.

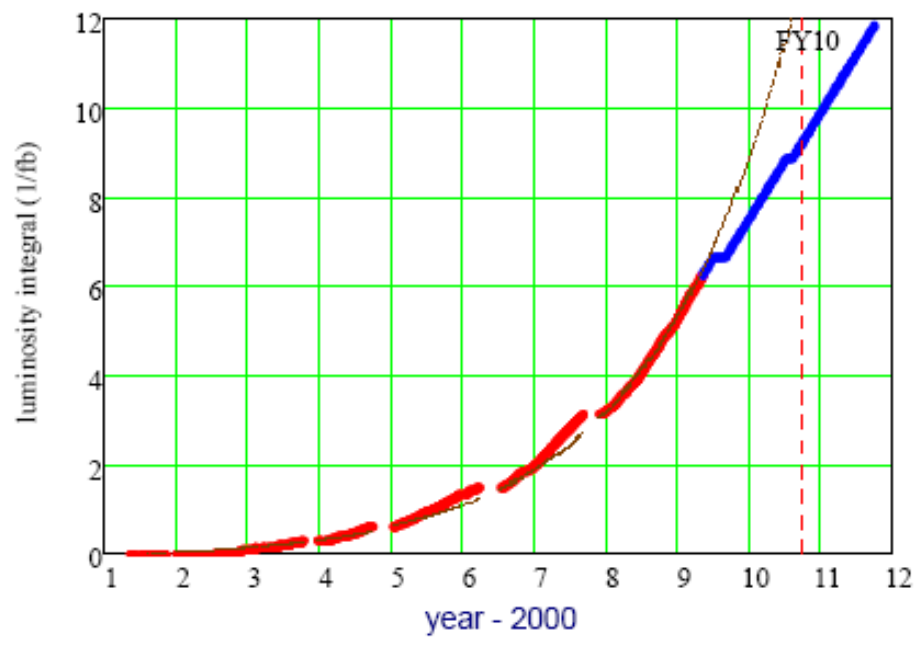

Figure 12. Tevatron Run II luminosity integral; red - actual, blue - projected, dashed line - an exponential growth with luminosity doubling every 17 months

The driver of luminosity growth during last three years has been the increase of antiproton production and the cooling optimization, together with operational improvements in the injection complex and the Tevatron itself.

Tevatron experience will be exploited by the next hadron collider: LHC will come into operation November this year (15). The first collisions will be done at the injection energy, 450 $\mathrm{GeV}$ per beam, and after one month the first data taking at $3.5 \mathrm{TeV}$ per beam is foreseen. During 2010 the energy will be increased up to $5 \mathrm{TeV}$ per beam, as the hardware systems and beam control are tested and commissioned. A shutdown period will follow to fully check and prepare all systems to go up to the nominal energy of $7 \mathrm{TeV}$. Thereafter the design luminosity of $10^{34} \mathrm{~cm}^{-2} \mathrm{sec}^{-1}$ should be reached.

LHC upgrade programs (16) have been developed for several years, and different options have been thoroughly investigated, in order to be ready to choose one when the LHC present configuration will reach its performance limits. They have been pursued, mostly in the framework of the FP6-CARE Project, in collaboration with US-LARP. Several of the initial scenarios have been abandoned, with the identification of show-stoppers.

Luminosity upgrades are mainly based on higher currents and smaller beams at the IP.

The first upgrade, already approved and in the construction phase, will substitute the linac in the injector chain with LINAC4, which will allow to increase the current in the collider by $40 \%$. By substituting the IR quadrupoles with large aperture ones, and adding new separation dipoles, all of them based on NbTi technology, the $\beta^{*}$ will be decreased from the present $55 \mathrm{~cm}$ 
to $30 \mathrm{~cm}$. Optimization of collimation schemes, together with compensation of the added chromaticity and the beta beating, is expected to raise the luminosity by a factor of two by 2014 .

More advanced designs of the IR quads, based on $\mathrm{Nb}_{3} \mathrm{Sn}$ technology, and more aggressive upgrade of the injector, whose details are still under discussion (new SC linac or a new PS2), are the main ingredients of a second upgrade stage, to be completed in about ten years from now, which should increase the luminosity in the range of $10^{35} \mathrm{~cm}^{-2} \mathrm{sec}^{-1}$. Adopting different collision schemes, some of which are based on the present lepton factories developments, is also envisaged as upgrading element.

High currents issues are being studied and investigated also in collaboration with other communities, as for example the electron cloud effect.

One of the main issues to reach the high luminosity is the high stored energy: for the nominal LHC parameters ( $7 \mathrm{TeV}$ and $0.58 \mathrm{~A}$ ) each beam corresponds to $400 \mathrm{MJ}$, two orders of magnitude higher than in the Tevatron. The LHC luminosity upgrade implies a further increase in stored beam energy by a factor $2-3$, which appears quite moderate compared with the step from the Tevatron to the nominal LHC. Tight beam control together with reliable and powerful collimation and beam dump systems are necessary.

Aiming at high flexibility in terms of accelerated ion species and colliding energies, $R H I C$ (35) is obtaining a series of excellent results, like the recent polarized proton collisions at 250 $\mathrm{GeV}$ and high luminosity $\left(1.210^{32} \mathrm{~cm}^{-2} \mathrm{sec}^{-1}\right)$, and $\mathrm{Au}-\mathrm{Au}$ collisions at low energies. These results have been obtained thanks to the developments in the understanding of hadron collider limiting factors, such as intrabeam scattering, electron cloud, beam-beam effects, magnet vibrations, and the efficiency of novel countermeasures such as bunched beam stochastic cooling, beam scrubbing and chamber coatings.

Ion collisions will be tested also in LHC, where already after few months from start up $\mathrm{Pb}$ collisions are foreseen.

\section{Lepton-hadron colliders}

Several projects of lepton-hadron colliders are being considered, both in USA and in Europe (36), mainly driven by the nuclear physics community interest. All of the HERA successor options are contemplating two choices: ring-ring (RR) and ring-linac (RL) configurations.

In USA the Electron-Ion Collider (EIC) initiative is considering eRHIC at Brookhaven National Laboratory using RHIC and ELIC at Jefferson Laboratory using CEBAF.

In $e$ RHIC RR proposal a $\sim 10 \mathrm{GeV}$ electron storage ring is added to the RHIC complex. Both polarized electrons and positrons are envisaged. The design luminosity is of the order of 3 $10^{32} \mathrm{~cm}^{-2} \mathrm{~s}^{-1}$. There are no significant R\&D issues associated with this concept which is therefore cost effective. The LR concept foresees a four time recirculated electron linac, and a slightly higher luminosity. 
ELIC is a more ambitious concept (37) based around the existing CEBAF at Jefferson Lab and would require a new ion complex, as shown schematically in Figure 13. Center-of-mass energy between $20 \mathrm{GeV}$ and $90 \mathrm{GeV}$ with an energy asymmetry of $\sim 10$, and several polarized ion species $\left(\mathrm{H}, \mathrm{D},{ }^{3} \mathrm{He}, \mathrm{Li}, \ldots\right)$ are considered.

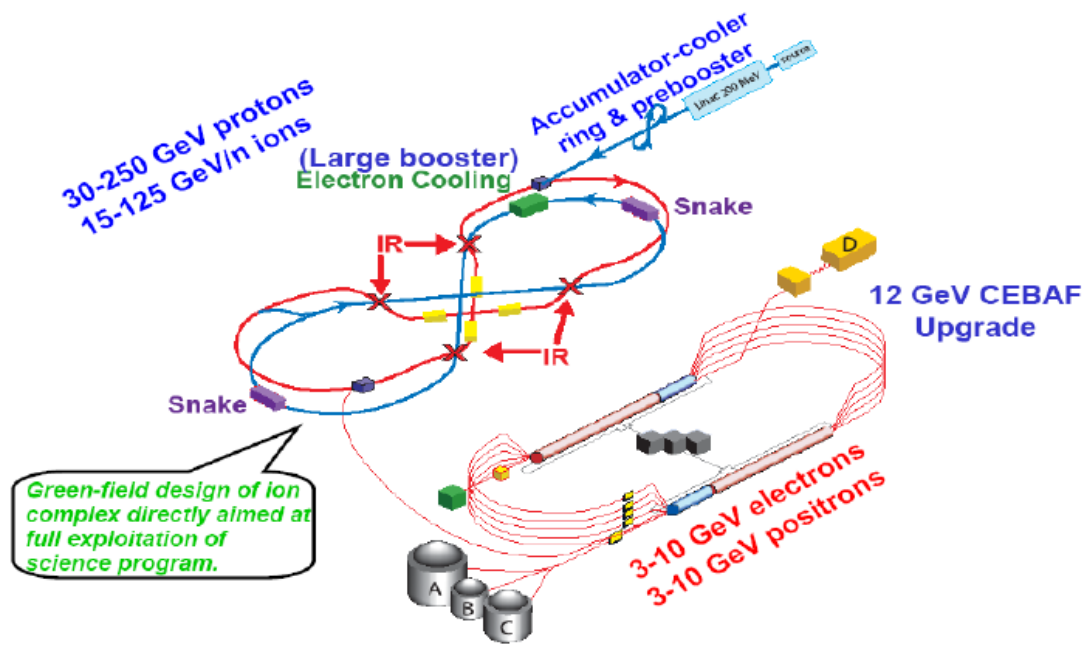

Figure 13. ELIC schematic layout at Jefferson Lab

This concept aims at a staged luminosity up to $10^{35} \mathrm{~cm}^{-2} \mathrm{~s}^{-1}$ accomplished in 4 IPs. A sophisticated 8-shaped ion ring intersects the electron ring, which is used also for the high energy cooling of the ion beams. CEBAF at $12 \mathrm{GeV}$ is used as injector. Very high collision frequencies, up to $500 \mathrm{MHz}$, crab crossing, beam polarization manipulation through IR design, ion beam high current stabilization, are some of the special features of this design.

$\mathrm{LHeC}$ (38) is one of the possible LHC upgrades. The RR option foresees a new electron ring inside LHC tunnel, similar to LEP, with a maximum energy of $80 \mathrm{GeV}$, and with a much higher circulating current (100 mA instead of $6 \mathrm{~mA})$. The electron beam size is matched to the size of the protons, as a smaller electron beam could have adverse effects on the proton beam lifetime. Design luminosity is in the range of $10^{33} \mathrm{~cm}^{-2} \mathrm{~s}^{-1}$, decreasing as the electron energy increases since constant power consumption is assumed. The electron beam would be injected by the new SC linac, SPL.

The linac for the RL option is similar to the XFEL and ILC, requiring a higher beam current than these two linacs and the energy range is $60-140 \mathrm{GeV}$. Operation as a recirculating linac is conceivable, opening the possibility of energy recovery from the spent beam after collision, which would boost luminosity at constant wall plug power.

Finally in the Electron-Nucleon Collider $(E N C)$ considered at GSI, a small electron ring would be built to provide $3 \mathrm{GeV}$ polarized electron beams to collide with the planned $15 \mathrm{GeV}$ HESR of FAIR, at a luminosity of $10^{33} \mathrm{~cm}^{-2} \mathrm{~s}^{-1}$. 


\section{Neutrino facilities}

The possibility of producing neutrino beams by using accelerator systems is being exploited in existing facilities or being considered for future ones, especially after the discovery that neutrinos are massive particles, that has implications from high-energy physics to cosmology. The core system of any neutrino facility is a HPPA, which stays for High Power Proton Accelerators. Under this common umbrella a vast variety of applications can be found, as Spallation Neutron Sources, production of radioactive beams, transmutation of nuclear waste, technological irradiation tools.

The maximum beam power produced in a HPPA today corresponds to SNS (39) in Oak Ridge, where almost $1 \mathrm{MW}$ has been recently reached. Spallation sources with similar or higher beam power will be built in China (CSNS) and Europe (ESS), this last in the recently decided site of Lund.

The initial state of any HPPA (40) are a high current ion source, an RFQ, a low energy Linac, which is usually a normal conducting structure (DTL, SDTL, CCDTL), up to the energy where SC linacs are more convenient (few hundreds of $\mathrm{MeV}$ ). Due to recent progress in low- $\beta$ SC cavities, like the spoke-cavities, some of the HPPA design start the SC part in lower energy region. Energies above few hundreds of $\mathrm{MeV}$ are always obtained with SC Linacs.

Neutrino beams from accelerators are or will be obtained through three different methods:

- Superbeams from pion decays

- Beta beams from radioactive ion decays

- Neutrino factories from muon decays

\section{Superbeams}

A high power proton beam, produced by a linac, or extracted from a synchrotron, is sent to a target, followed by magnetic horns and reflectors which focus the charged secondaries, and by a decay tunnel, where neutrinos are produced by pion decays and directed toward the detectors, usually a nearby detector for the first characterization of the starting beam and a detector placed at hundreds $\mathrm{km}$ for the neutrino oscillation detection.

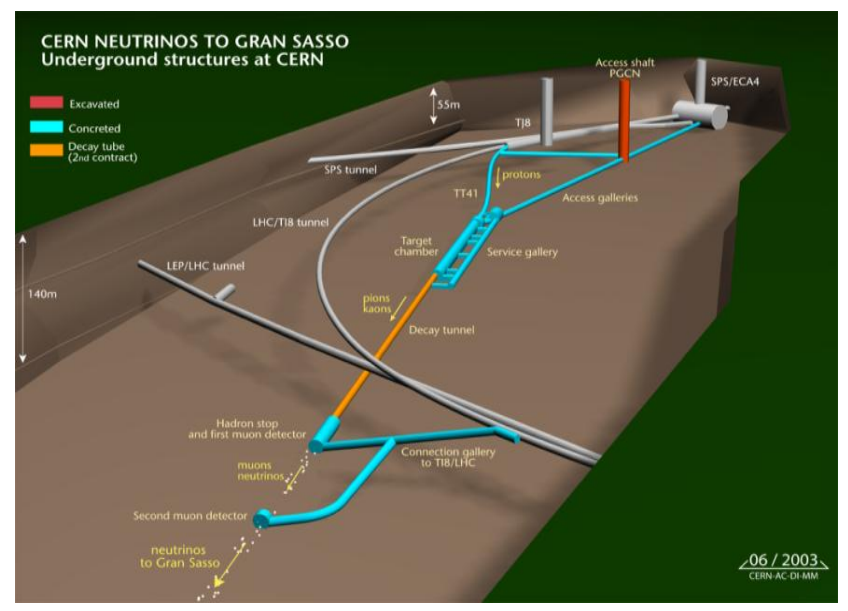

Figure 14. Artistic view of CNGS layout at CERN 
Presently three facilities are active in the world, KEK PS in Japan, NUMI at Fermilab and CNGS in Europe. Their characteristic POT (Protons On Target) is measured in few $10^{19} /$ year. As an example Figure 14 shows the CERN site of CNGS, which delivers neutrinos to the underground Gran Sasso Laboratory in Italy, after travelling a distance of $730 \mathrm{~km}$, the optimum distance for detecting the oscillation of the $17 \mathrm{GeV}$ neutrinos.

Future projects are based on a much more intense source of protons, which should rise the POT/year up to $10^{21}$ and distance source-detector of thousands $\mathrm{km}$.

Fermilab for example is considering for its future high intensity projects the possibility of two new neutrino baselines, NoVA and DUSEL (41), which would profit from the intensity upgrade of the Main Injector, through the proposed Project-X.

At CERN, exploiting one of the possible LHC upgrade scheme, the possibility of using the proton beam of the sc linac SPL for a more intense neutrino production is being studied.

\section{Beta Beams}

Another method for the production of intense and pure neutrino beams is the "beta-beam" (41). It exploits boosted radioactive ions decaying through beta-decay. Protons impinge on a target to produce radioactive species of interest, using an ISOL target. After ionization and bunching, the ions are accelerated in different stages up to an energy of $100 \mathrm{GeV} / \mathrm{A}$, as in the production of very intense radioactive beams (EURISOL), and finally stacked longitudinally in a decay ring. A sketch of the beta-beam facility proposed for CERN is shown in Figure 15.

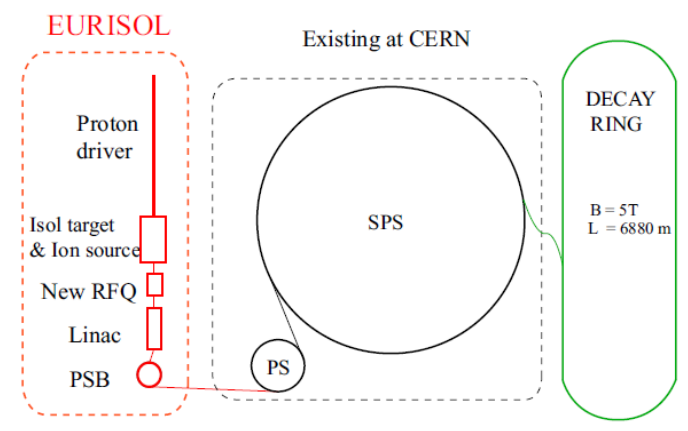

Figure 15. Schematic layout of beta-beam complex proposed at CERN

\section{Neutrino factories and muon colliders}

Neutrino factories would be the most powerful neutrino sources based on accelerators (43). A high power (few MW) proton beam is again at the basis of the complex. It is sent to a target, usually liquid $\mathrm{Hg}$, where pions are produced. They decay into muons, whose phase space is cooled by ionization. They are then accelerated to energies of tens of $\mathrm{GeV}$, and finally stored in storage rings, with long straight decaying sections. From these sections the neutrino beams are directed through the earth to the detectors, placed in underground laboratories, screened 
from the cosmic rays. Racetracks or triangular shapes can be used for the decaying rings according to the number and position of detectors (see Figure 16)

A natural evolution of a neutrino factory is the muon collider by substituting the decay ring with a collider ring, and with a more powerful muon cooling to assure the small dimensions at collision. Indeed all groups involved in such R\&D are studying both projects.

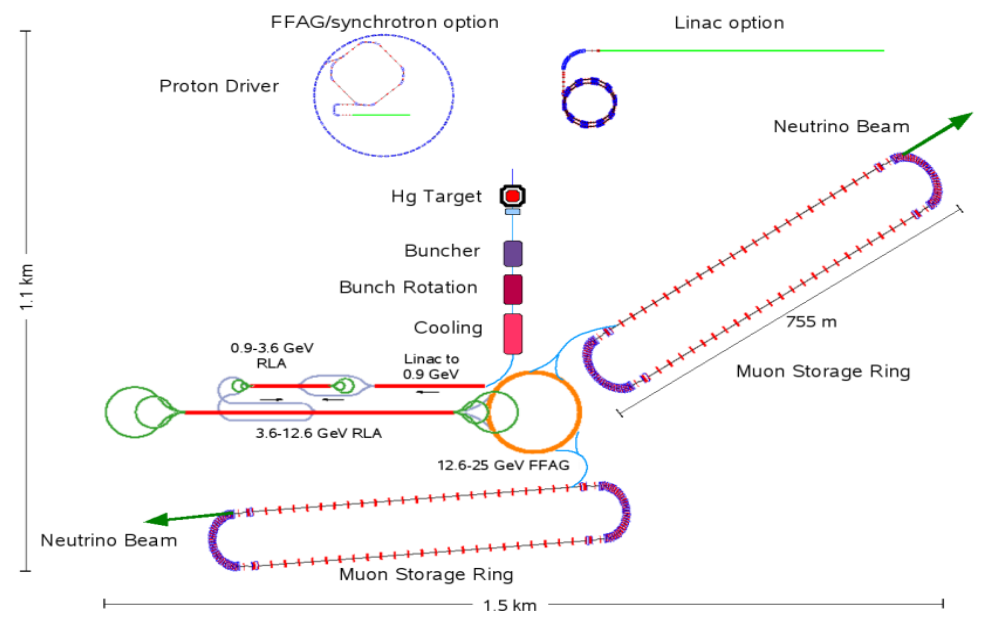

Figure 16. Schematic layout of a neutrino factory

A Neutrino Factory or a Muon Collider will be very large investment, that can be addressed only under international collaborations. Presently the ISS (International Scoping Study) for a Future Neutrino Factory and Superbeam Facility design study has been formed. The main goal of the activity is to reach consensus on a baseline design for a Neutrino Factory complex, including proton driver parameters, choice of target, front-end design, acceleration system design, and decay ring geometry. Another goal is to explore the common features, if any, between the proton driver for a Neutrino Factory and those for a Superbeam or Beta Beam facility. In general, the requirements for either of the latter facilities are less stringent than those for a Neutrino Factory.

Test facilities are being or will be operated to study in detail some of the key issues of such complexes: MERIT (MERcury Intense Target) (44) at CERN is a proof-of-principle demonstration of a free mercury jet target for a 4-MW proton beam. MICE (Muon Ionisation Cooling Experiment) (45) at RAL in UK, is proving the muon cooling techniques. EMMA (Electron Model of Muon Acceleration) (46) at the CCLRC in Daresbury should demonstrate the principle of non-scaling FFAGs (Fixed Field Alternate Gradient) for muon acceleration.

\section{Plasma accelerators}

Developments in colliders aim at next future realization, neutrino factories and muon colliders could be reality not before twenty years from now. In this section a brief look at the still further future will be given.

Plasma acceleration, driven by lasers or by electron beams, has been the subject of studies and tests since several decades. Recently tremendous progresses on the achievable energy 
gradient has been obtained (see Figure 17). At SLAC, thanks to the production of brilliant electron bunches, accelerating gradients of $\sim 50 \mathrm{GeV} / \mathrm{m}$ have been measured: a $45 \mathrm{GeV}$ electron beam has doubled its energy after $1 \mathrm{~m}$ interaction with the plasma.

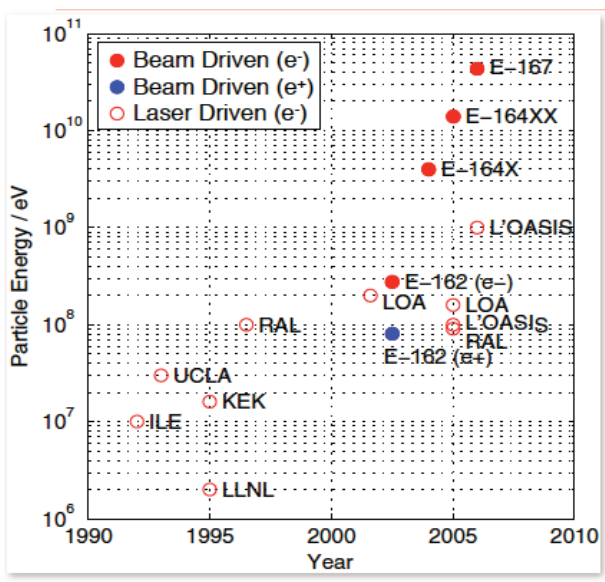

Figure 17. Accelerating gradients in plasma wake field acceleration

Results are so encouraging that a collaboration has been formed with the aim of investigating the possibility of designing a linear collider in the $10 \mathrm{TeV}$ center of mass energy range based on plasma acceleration.

The accelerating gradient would be created by a drive beam, similar to the CLIC one. The gradient of the main linac should reach $25 \mathrm{GeV} / \mathrm{m}$.

In order to test all issues of such an idea, a test facility, FACET (47) (Facilities for Accelerator Science and Experimental Test), is being built at SLAC, where the first two-thirds of the linac will be used to bring electron and positron beams to a new experimental area, just upstream the LCLS injector. In addition, the last 200 meters of the PEP-II injection line would be re-routed to bring a lower energy electron beam from the linac into End Station. In one of the early experiments, researchers will split the compressed electron bunches into two parts, using the first bunch to ionize and drive the plasma that will in turn accelerate the trailing or "witness bunch." They will also be able to study acceleration of positively charged positron beams, which would interact differently with the negatively charged plasma.

The goal is to accelerate particles in a much shorter distance, making giant accelerators a thing of the past.

\section{Conclusions}

During the last five decades high energy physics quest for colliding beams with ever increasing energies has driven technology developments in the accelerator fields, which have thereafter spread in other fields for fundamental and applied physics.

Colliders have evolved from small projects, developed in national laboratories, to very complex systems, implying large investments in terms of costs and personnel, where 
participation of international teams is mandatory: international collaboration and wide exchange of ideas and results is the usual way of advancing in accelerator R\&D.

Activity in the accelerator community dedicated to HEP is such a wide field that a whole review would need much more than a conference paper. A facile overview has been done, and many interesting issues and details have been neglected or just mentioned, trying to balance the shortage by writing down a rich reference list, where detailed information can be found.

The next great appointment in HEP is the LHC start up. Many projects are being proposed for the next future: Linear Colliders, new Lepton Factories or upgrades of the existing ones, lepton-hadron colliders, neutrino facilities, muon colliders. Which of these projects will become reality will be defined once the first LHC results will show what is the best path to be followed.

\section{Acknowledgments}

During the preparation of my talk I have contacted several colleagues from many laboratories around the world, asking them to provide me information and material about the work they are doing. I will not mention all of them here, but they are included in the references list. I want to thank all of them for kindly sharing their knowledge, and for contributing to this review through my writing.

\section{References}

1. C.Bernardini, G.F.Corazza, G.Ghigo, B.Touschek. "The Frascati Storage Ring". Nuovo Cimento 18, 1293 (1960). 1960.

2. LHC. [Online] http://lhc.web.cern.ch/lhc/.

3. Tevatron. [Online] http://www.fnal.gov/pub/science/accelerator/.

4. RHIC. [Online] http://www.bnl.gov/rhic/.

5. VEPP 4M. [Online] http://v4.inp.nsk.su/index.en.html.

6. R. Assman, et al. "LEP Operation and Performance with $100 \mathrm{GeV}$ ". EPAC 00 Proceedings, Wien. 2000.

7. ILC. [Online] http://www.linearcollider.org/cms/.

8. CLIC. [Online] http://clic-study.web.cern.ch/CLIC-Study/.

9. TESLA. [Online] http://tesla-new.desy.de/.

10. S. Schreiber, et al. "FLASH Operation as an FEL User Facility". PAC 09 Proceedings, Vancouver. 2009.

11. XFEL. [Online] http://xfel.desy.de/.

12. A. Sessler, S. Yu. "Relativistic Klystron two-beam accelerator". Phys. Rev. Lett. 1987, Vol. 58, 23.

13. R. Corsini, et al. "Two-Beam Linear Colliders - Special Issues". PAC09 Proceedings, Vancouver. 2009. 
14. A. Valishev, et al. "Recent Tevatron Operational Experience". PAC 09 Proceedings, Vancouver. 2009.

15. L.Evans. "LHC status". This conference. 2009.

16. J.-P. Koutchouk, F. Zimmermann. "LHC Upgrade Scenarios". PAC 09 Proceedings, Vancouver. 2009.

17. VLHC. [Online] http://vlhc.org/.

18. G.Sabbi. "15 T and Beyond - Dipoles and Quadrupoles". WAMSDO 2008 Proceedings. http://indico.cern.ch/conferenceDisplay.py?confId=28832, 2008.

19. M.E.Biagini, Editor. ICFA BD Newsletter No. 48. [Online] http://wwwbd.fnal.gov/icfabd/Newsletter48.pdf.

20. C. Zhang, et al. "Construction and Commissioning of BEPC-II". PAC O9 Proceedings, Vancouver. 2009.

21. G. Vignola, et al. "DAFNE, The Frascati F-factory". PAC93 Proceedings, 1993. 1993.

22. M. Zisman, et al. "PEP II". PAC 99 Proceedings, New York. 1999.

23. High-Energy-Accelerator-Research-Organization. "KEKB B-factory Design Report". KEK Report 95-7. 1995.

24. Yu.M.Shatunov, et al. "Project of a New Electron-Positron Collider VEPP-2000". EPAC OO Proceedings, Wien. 2000.

25. P.Raimondi. "Crab waist collisions in DAFNE and SuperB Design". EPACO8 Proceedings. 2008.

26. F. Ruggiero, F. Zimmermann. "Luminosity optimization near the beam-beam limit by increasing bunch length or crossing angle". Phys. Rev. ST-AB 5. 2002.

27. M.Bone, et al. "SuperB: A High Luminosity Asymmetric e+e-Super Flavour Factory. CDR". eprint: ArXiv:0709.0451. INFN/AE-07/2, SLAC-R-856. 2007.

28. A. Blinov, et al. "The Project of T-Charm Factory With Crab Waist in Novosibirsk". Proceedings of 40th ICFA ABDW 2008, Novosibirsk, Russia. 2008.

29. K. Oide, et al. "A Few Issues On The Upgrade OF KEKB B-Factory". PAC 09 Proceedings, Vancouver. 2009.

30. J.Seeman, et al. "Last Year of PEP-II B-Factory Operation". EPAC 08 Proceedings, Genoa. 2008.

31. CesrTA. [Online] https://wiki.lepp.cornell.edu/ilc/bin/view/Public/CesrTA/.

32. ATF. [Online] http://atf.kek.jp/.

33. Y. Honda, et al. "Achievement of Ultralow Emittance Beam in the Accelerator Test Facility Damping Ring". Phys.Rev.Lett., 92, 054802 . 2004.

34. A. Seryi, et al. ATF2 COMMISSIONING. PAC 09 Proceedings, Vancouver. 2009.

35. C.Montag. "RHIC Progress And Future". PAC 09 Proceedings, Vancouver. 2009.

36. R.Milner. "Overview Of Electron-Ion Collider Initiatives". PAC 09 Proceedings, Vancouver. 2009. 
37. S.A. Bogacz, et al. Advances on ELIC Design Studies. EPAC 08 Proceedings, Genoa. 2008.

38. F. Zimmermann, et al. "The Large Hadron-Electron Collider (LHEC) at the LHC". PAC 09 Proceedings, Vancouver. 2009.

39. J. Galambos, SNS Team. "Progress with MW-Class Operation of the Spallation Neutron Source". PAC 09 Proceedings, Vancouver. 2009.

40. J.M.Lagniel. "LINAC Architecture for High Power Proton Sources". XX International Linac Conference, Monterey. 2000.

41. E.Prebis. "Life After the Tevatron: The Intensity Frontier at Fermilab". This conference. 2009.

42. C.Volpe. "Topical Review on "Beta-beams". [Online] 2006. http://arxiv.org/abs/hepph/0605033v2. hep-ph/0605033.

43. M. Apollonio, et al. "Accelerator design concept for future neutrino facilities". [Online] 2007. http://www.cap.bnl.gov/mumu/project/ISS/ISS-AcceleratorWG-final-submitted-R1.pdf.

44. MERIT. [Online] http://proj-hiptarget.web.cern.ch/proj-hiptarget/.

45. MICE. [Online] http://hepunx.rl.ac.uk/uknf/mice/.

46. T.R.Edgecock. "EMMA - the World's First Non-scaling FFAG". PAC 07 Proceedings, Albuquerque. 2007.

47. M.Hogan. "Plasma Wakefield Accelerator Experiments at the FACET ASF". PAC O9 Proceedings, Vancouver. 2009. 